\title{
Transition in the Outflow Evolution of the Massive Star-forming Region W75N
}

\author{
Jeong-Sook Kim* \\ National Astronomical Observatory of Japan, Japan \\ E-mail: jskim@nao.ac.jp
}

\section{Soon-Wook Kim}

Korea Astronomy and Space Science Institute, Republic of Korea

Korea University of Science and Technology, Republic of Korea

E-mail: skim@kasi.re.kr

\begin{abstract}
W75N is one of the representative massive star forming region and very bright $\mathrm{H}_{2} \mathrm{O}$ maser source at $22 \mathrm{GHz}$. Although they share the same environment in W75N, VLA1 and VLA2 have been detected with different $\mathrm{H}_{2} \mathrm{O}$ maser features in 1999 and 2005 VLBA observations: jet-like and wind-like outflows, respectively. In 2007, we found, for the first time, that the outflow of VLA 2 has a transition from a shell-like to more elongated jet-like outflow, with the VLBI Exploration of Radio Astrometry (VERA), a Japanese facility of the Very Long Baseline Intermetry (VLBI). We show the acceleration of the outflow as a result of the transition. Previously, such transition and acceleration have never been explicitly observed. Our result is consistent with a recent magnetohydrodynamic simulations. In 2012, the Very Long Baseline Array (VLBA) polarization observation have confirmed a further expansion of the elongated structure in VLA 2. Furthermore, the orientation of the magnetic field in VLA 2 has been changed a new direction along the major axis of the outflow, and became aligned with that in VLA 1. All these observations strongly support the transition from a circular, uncollimated to a more elongated, collimated outflow first identified in the 2007 VERA observations.
\end{abstract}

12th European VLBI Network Symposium and Users Meeting,

7-10 October 2014

Cagliari, Italy

\footnotetext{
* Speaker.
} 


\section{Introduction}

It is a matter of debate how the process of massive star formation connects with its outflow evolution. In the very early stages of high mass star formation, the well-collimated outflows could first occur and, later on, the outflows become less collimated due to the formation of an HII region (e.g., Beuther \& Shepherd 2005). On the contrary, recent magnetohydrodynamic (MHD) simulations can produce that poorly collimated outflows in the very early stage of massive star formation can be form depending on magnetic field strength associated with their protostellar disks (e.g., Seifried et al. 2012).

The Very Long Baseline Interferometry (VLBI) observations of $\mathrm{H}_{2} \mathrm{O}$ at $22 \mathrm{GHz}$ have played an important role to trace the outflows and accretion disks in the very early stage of the star formation. However, the number of observations are still not enough.

$\mathrm{W} 75 \mathrm{~N}(\mathrm{~B})$ is a massive star-forming region located in the Cygnus $\mathrm{X}$ complex of dense molecular clouds at a distance of $1.3 \mathrm{kpc}$ (Rygl et al. 2012). Three massive young stellar objects, VLA 1, VLA 2, VLA 3 are identified located within $\sim 2000 \mathrm{AU} \times 2000 \mathrm{AU}$ (Torrelles et al. 1997), probably early B spectral types (e.g., Shepherd et al. 2004; Carrasco-González et al. 2010). VLA 1 and VLA 3 display elongated radio continuum, consistent with a thermal radio jet. On the contrary, the unresolved continuum emission is observed in VLA 2, located between VLA1 and VLA2. Due to their proximity, the three sources are thought to be at different evolutionary stages from each other (Torrelles et al. 1997; Carrasco-González et al. 2010).

Remarkably different geometries of outflows in VLA 1 ans VLA 2 were identified with the multi-epoch VLBI observations of $22 \mathrm{GHz} \mathrm{H}_{2} \mathrm{O}$ masers performed with the Very Long Baseline Array (VLBA) in 1999 (Torrelles et al. 2003). The collimated jet-like structure of $\mathrm{H}_{2} \mathrm{O}$ masers

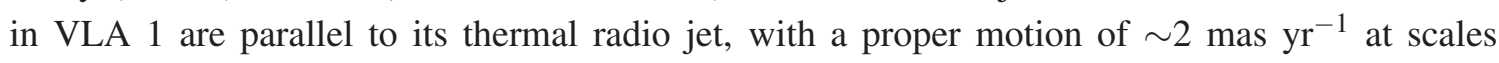
of $\sim 1300$ AU. On the other hand, the $\mathrm{H}_{2} \mathrm{O}$ masers in VLA 2 show a almost circular shell-like structure of $\sim 150 \mathrm{AU}$ around the central source, moving outward in multiple direction. The result was surprising and contradicts to a typical scenario of the outflow evolution in massive protostars, initially from a collimated to uncollimated outflow. Torrelles et al. (2003) suggest that VLA 2 could be in an earlier stage than VLA 1 from the fact that the observed uncollimated motions around VLA 2 are smaller in scale compared with the collimated motions around VLA 1. The result is surprising because the usual belief has been that outflows start with very collimated ones and become less collimated with age (e.g. Beuther \& Shepherd 2005).

\section{Evolution of Expanding Maser Shell in VLA 2}

After the first VLBI observations in 1999, follow-up observations have been carried out: a single epoch in 2005 with VLBA (Surcis et al. 2011), three-epochs in 2007 with VERA (Kim et al. 2013), and a single-epoch in 2012 with EVN (Surcis et al. 2014), respectively. The spatial distribution of the observed $\mathrm{H}_{2} \mathrm{O}$ masers with VERA in VLA 1 and VLA 2 is presented in Figure 1 (also see Kim et al. 2013). As shown in Figure 1, all VLBI observations since 1999 show that $\mathrm{H}_{2} \mathrm{O}$ masers were detected in VLA 1 and VLA 2, but no maser was detected in VLA 3. The apparent distribution in 2007 looks similar to that previously observed with VLBA in 1999 (Torrelles et al. 2003) and 2005 (Surcis et al. 2012): a linear jet-like structure in VLA 1 and a shell-like structure 


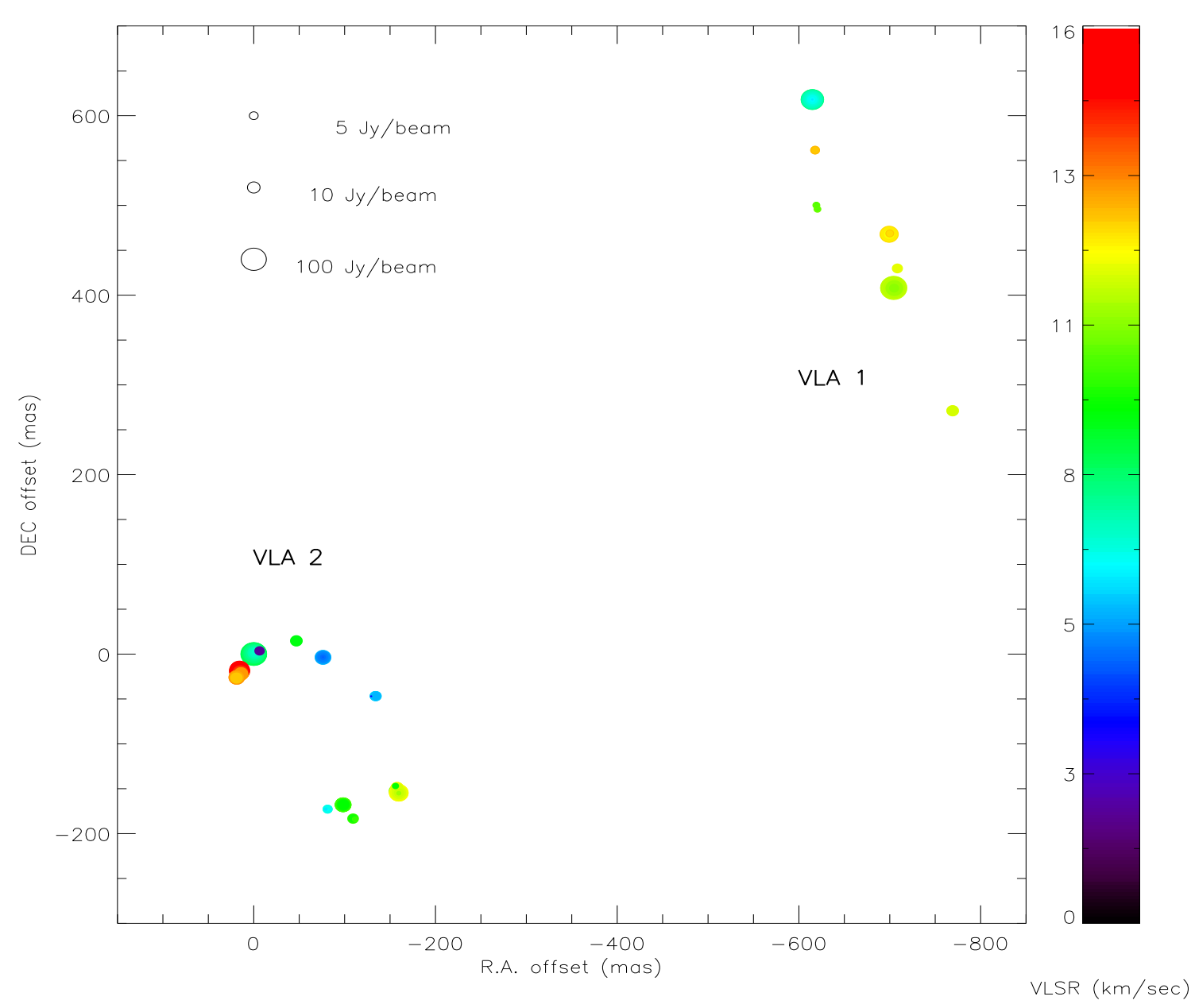

Figure 1: Position of the $\mathrm{H}_{2} \mathrm{O}$ maser spots (filled circles) observed with VERA at $22 \mathrm{GHz}$ in 2007 around VLA 1 and VLA 2 (indicated by plus symbols). The filled circle symbols of the masers are scaled logarithmically according to their peak flux density. Open circle symbols for 5, 10, and $100 \mathrm{Jy} \mathrm{beam}^{-1}$ are shown in the upper left corner of the first panel. The color of the symbols are codified by their radial velocity. The offset positions are relative to the reference maser spot position $(0,0)$ used for self-calibrating the data (for further discussion, see Kim et al. 2013).

in VLA 2 in all three epochs. In particular, the size and shape of VLA 2 have changed (see Figure 2 and the next section).

\section{Transition of Outflows in VLA 2 observed in 2007 with VERA}

To perform a further detailed comparison of our observations in 2007 and 2012 to the previous VLBI observations in 1999 and 2005, we performed elliptical fits to the $\mathrm{H}_{2} \mathrm{O}$ maser position detected in 1999 and 2005. To investigate further the evolution of the outflow in VLA 2, we plot the expansion size of VLA 2 in Figure 2. (also Kim et al. 2013; Surcis et al. 2014). The result is surprising. While the $\mathrm{H}_{2} \mathrm{O}$ maser features in VLA 1 and VLA 2 observed in 2005 (Surcis et al. 


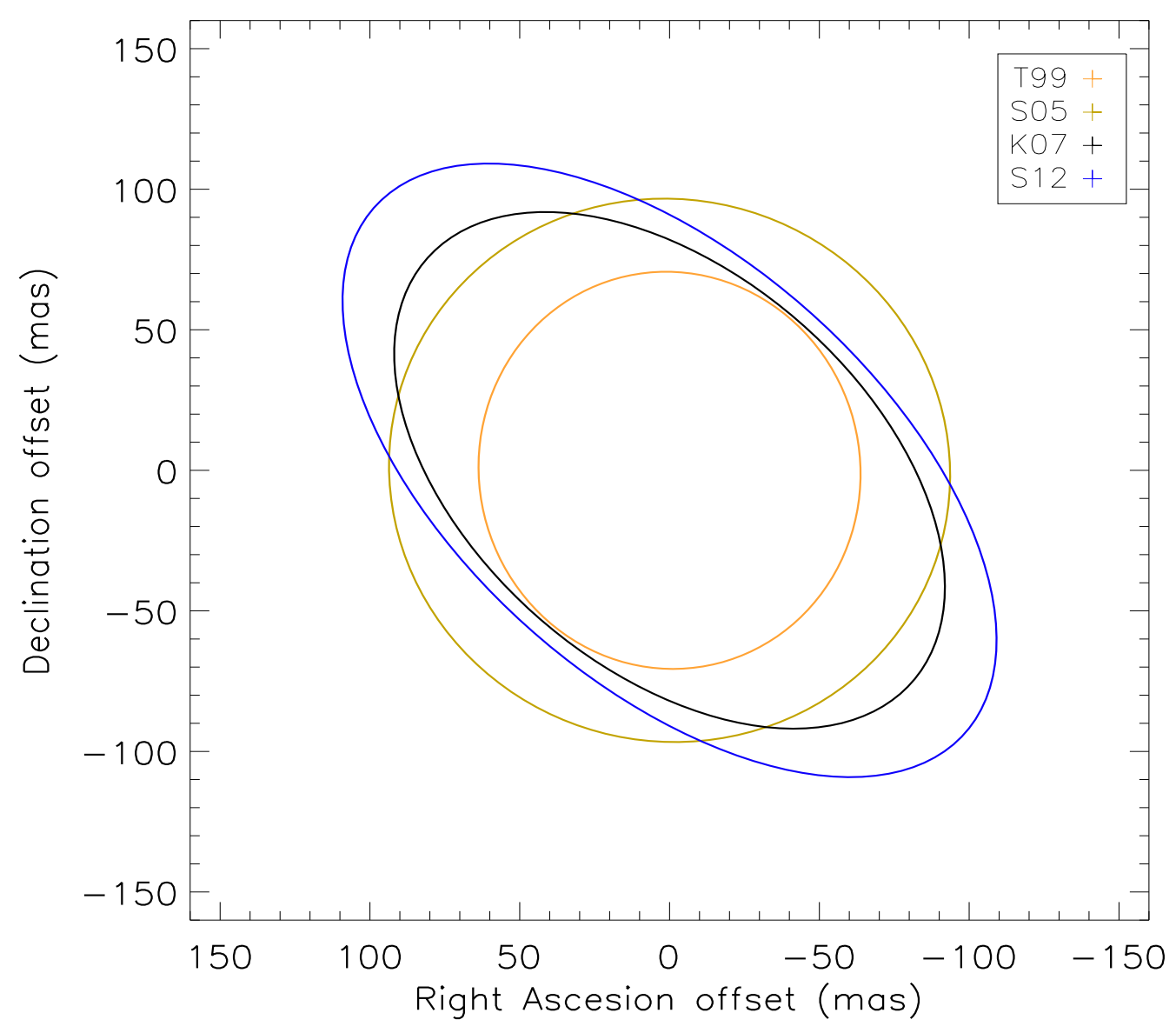

Figure 2: The elliptical model fits for the expanding $\mathrm{H}_{2} \mathrm{O}$ maser shell observed in 1999, 20052007 and 2012.

2011) were not appreciably different from the masers observed in 1999 (Torrelles et al. 2003), in 2007, the distribution became more elliptical. A further elliptical expansion went on in 2012.

In Figure 3, we present the evolution of the change in the major axis (Kim et al. 2013; Surcis et al. 2014). As shown in Figure 3, the expansion velocity in VLA 2 has not been constantly increased. The expanding shell in VLA 2 has suffered an acceleration at least for 2005-2007 as the shell evolves from a circular to more elongated distribution. Therefore, there might be a sudden episodic ejection event, or a change in the magnetic field strength associated with the protostellar disk. A possibility of an episodic ejection was discussed by Torrelles et al. (2003). The possibility of the global evolution of the magnetic field strength in the early stage of the massive star formation associated with a sub-Keplerian and Keplerian disks is first reproduced by recent MHD simulations (Seifried et al. 2012). A slow uncollimated outflow can be formed due to the magnetic pressure in the very early stage of the protostellar evolution. Once the Keplerian disk forms, a short-lived fast, collimated outflow overtakes the slow uncollimated outflow. Therefore, the 2007 VERA observations (Kim et al. 2013) may provide the first observational evidence for such an event. 


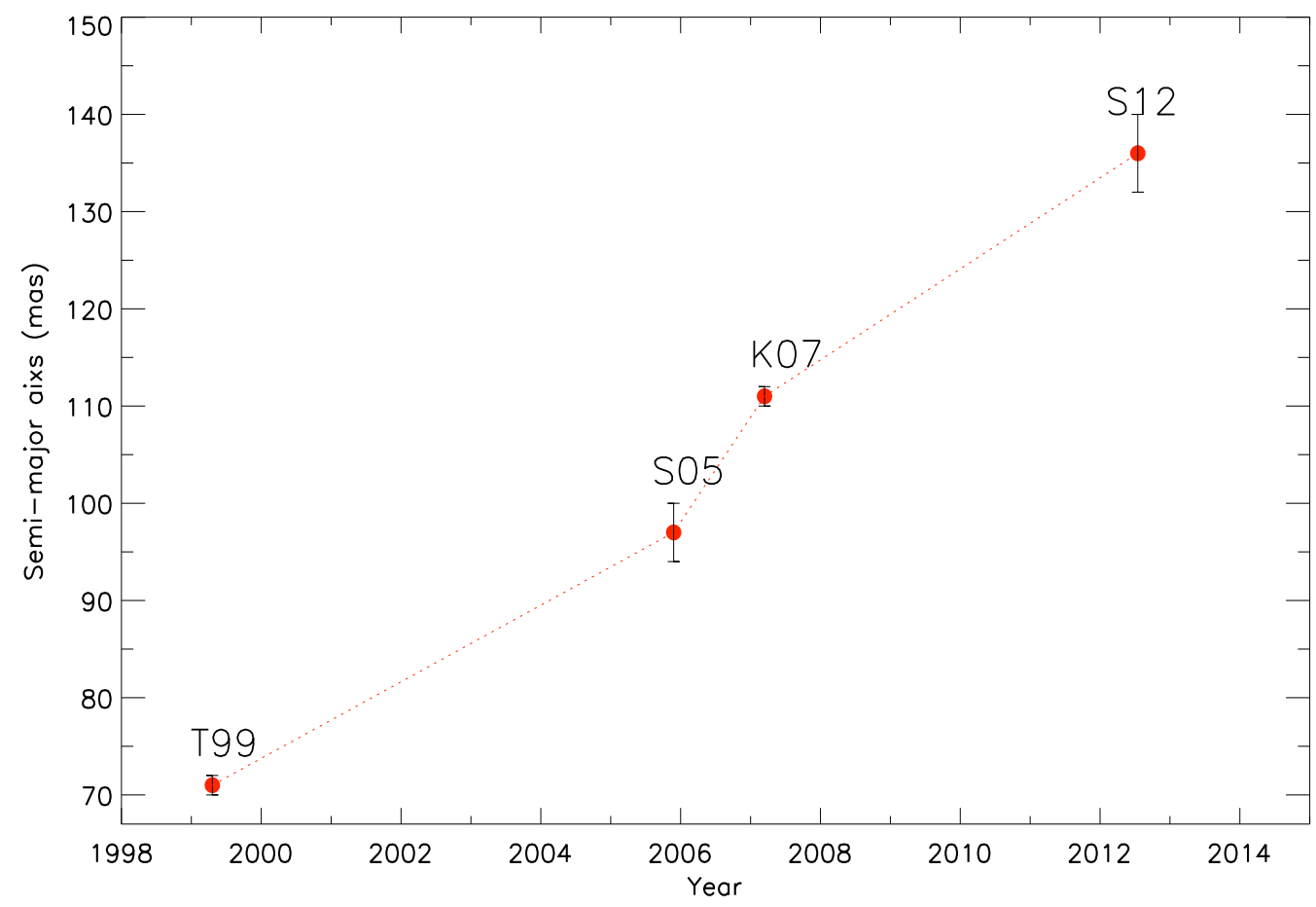

Figure 3: The change of the size of semi-major axis based on the elliptical fits to the expanding $\mathrm{H}_{2} \mathrm{O}$ maser shell in VLA 2 observed in 1999, 2005, 2007 and 2012 (Kim et al. 2013; Surcis et al. 2014).

\section{Polarization Observations of $\mathrm{H}_{2} \mathrm{O}$ Masers in 2012 with EVN}

The 2005 VLBA and 2012 EVN observation with polarization (Surcis et al. 2014) show that the $\mathrm{H}_{2} \mathrm{O}$ maser distribution and the magnetic field in VLA 1 have not changed for $\sim 7$ years since 2005. On the contrary, long with the dramatic change of its ellipticity in the expanding shell, the magnetic field strength estimated in the 2012 observation is about one third of that in 2005: 128 and $345 \mathrm{mG}$, respectively. We speculate that the change might be due to the launching of the fast outflow, as first identified in Kim et al. (2013). Furthermore, the orientation of the magnetic field in VLA 2 has changed its direction along the "new" major axis of the shell-like structure, and became aligned with the magnetic field in VLA 1. Therefore, all these changes strongly support the transition of outflow from a circular, uncollimated to a more elliptical, more collimated $\mathrm{H}_{2} \mathrm{O}$ maser shell in VLA 2 first identified with the 2007 VERA observations.

\section{References}

[1] Beuther, H., \& Shepherd, D. 2005, Cores to Clusters: Star Formation with Next Generation Telescopes, 105

[2] Carrasco-González, C., Rodríguez, L. F., Torrelles, J. M., Anglada, G., \& González-Martín, O. 2010, AJ, 139, 2433

[3] Kim, J.-S., Kim, S.-W., Kurayama, T., et al. 2013, ApJ, 767, 86 
[4] Seifried, D., Pudritz, R. E., Banerjee, R., Duffin, D., \& Klessen, R. S. 2012, MNRAS, 422, 347

[5] Shepherd, D. S., Kurtz, S. E., \& Testi, L. 2004, ApJ, 601, 961

[6] Surcis, G., Vlemmings, W. H. T., van Langevelde, H. J., et al. 2014, A\&A, 565, L8

[7] Surcis, G., Vlemmings, W. H. T., Curiel, S., et al. 2011, A\&A, 527, 48

[8] Torrelles, J. M., Gómez, J. F., Rodríguez, L. F., Ho, P. T. P., Curiel, S., \& Vázquez, R. 1997, ApJ, 489, 744

[9] Torrelles, J. M., Patel, N. A., Anglada, G., et al. 2003, ApJ, 598, L115 